\title{
Diagnóstico de la Educación para el Desarrollo en áreas rurales: un análisis correlacional de las actitudes de la población europea
}

\author{
Noelia Santamaría-Cárdaba', José M. Marbán Prieto², Luis \\ Torrego Egido ${ }^{3}$
}

Resumen: El proyecto Rural DEAR Agenda EYD-20154 planteaba, como principal propósito, promover un cambio de actitudes sociales que impulsara un desarrollo más sostenible y humano, mediante una propuesta de acciones y estrategias inclusivas para sensibilizar y educar para la ciudadanía global en municipios rurales europeos. La presente investigación profundiza en el perfil actitudinal de la población europea participante en el mencionado proyecto mediante un análisis correlacional de las respuestas recogidas por el mismo. Los resultados nos permiten identificar relaciones entre respuestas y categorías de respuestas que ayudan a comprender el comportamiento de la ciudadanía rural europea con respeto a la Educación para el Desarrollo. Al mismo tiempo, los resultados abren la puerta a nuevos análisis de tipo causal o predictivo que favorezcan el desarrollo de propuestas de intervención más eficientes en las zonas involucradas en el estudio.

Palabras clave: Análisis correlacional, educación para el desarrollo, opinión pública, actitudes, rural.

\footnotetext{
1 Universidad de Valladolid. Departamento de Sociología y Trabajo Social. Contratada predoctoral FPU. Doctoranda en Investigación Transdisciplinar en Educación.

${ }^{2}$ Universidad de Valladolid. Profesor Titular de Universidad. Departamento de Didáctica de las Ciencias Experimentales, Sociales y de la Matemática. Doctor por la Universidad de Valladolid.

${ }^{3}$ Universidad de Valladolid. Profesor Titular de Universidad. Departamento de Pedagogía. Doctor por la Universidad Nacional de Educación a Distancia.

${ }^{4}$ Project funded by European Commission - EuropeAid/134863/C/ACT/Multi.
} 
Fecha de recepción: 9 de febrero de 2019.

Fecha de admisión definitiva: 12 de noviembre de 2019.

\section{Diagnosis of Development Education in rural areas: a correlational analysis of the attitudes of the European population}

Abstract: The project Rural DEAR Agenda EYD-2015 established as its main purpose to promote a change in social attitudes that drives a more sustainable and humane development, through a proposal of inclusive actions and strategies to raise awareness and educate for global citizenship in rural European municipalities. This article aims to deepen the attitudinal profile of the participating European population through correlational analysis of the responses collected by the mentioned project. The results allow us to understand the behavior of the European rural population with respect to the Education for Development. Al the same time, the results obtained open the door to future analysis of causal or predictive type in order to ease the development of more effective intervention actions within the participating rural areas.

Key words: Correlational analysis, education for development, public opinion, attitudes, rural.
Diagnostique de l'Education au Développement dans les zones rurales: une analyse corrélationnelle des attitudes de la population européenne

Résumé: L'objectif principal du projet Rural DEAR Agenda EYD-2015 était de promouvoir un changement $d^{\prime}$ attitudes sociales qui favoriserait un développement plus durable et plus humain, grâce à une proposition $d$ 'actions et de stratégies inclusives pour sensibiliser et éduquer à la citoyenneté mondiale dans les municipalités rurales européennes. La présente recherche approfondit le profil attitudinal de la population européenne participant au projet mentionné au moyen d'une analyse corrélationnelle des réponses recueillies par le projet. Les résultats nous permettent $d$ 'identifier les relations entre les réponses et les catégories de réponses qui aident à comprendre le comportement des citoyens ruraux européens en matière d'éducation au développement. En même temps, les résultats ouvrent la porte à de nouvelles analyses causales ou prédictives qui favorisent l'élaboration de propositions $d^{\prime}$ intervention plus efficaces dans les domaines visés par l'étude.

Mots clé: Analyse corrélationnelle, éducation pour le développement, opinion publique, attitudes, développement rural. 


\section{Introducción}

La Educación para el Desarrollo (en lo sucesivo, ED) es un proceso educativo abierto y dinámico que pretende desarrollar en las personas tanto su autonomía, como su conciencia crítica para que comprendan los problemas que afectan a la realidad social y a las relaciones Norte-Sur (Argibay y Celorio, 2005; Celorio, 2013). Su finalidad es crear una ciudadanía global que esté comprometida con los Derechos Humanos y que intente trasformar el mundo para convertirlo en un lugar más equitativo, justo y solidario. Ante esto no cabe duda de la relevancia de educar para el desarrollo en la sociedad actual, pues tiene un propósito primordial al buscar conseguir una mejora social que transforme el mundo en un lugar más humano. En este sentido, la ED persigue varios objetivos, destacando los dirigidos a sensibilizar a la sociedad para que comprenda los problemas relacionados con las desigualdades sociales y desarrolle una conciencia global que impulse un Desarrollo Sostenible y Humano.

La ED se desarrolla, como otros tipos de educación, preferentemente en zonas urbanas, y está pensada y organizada con una mentalidad urbana; no sólo porque la educación que llega a la población rural tenga una calidad baja, como sostiene Ekanayake, "y esa baja calidad repercute en la mala utilización de los recursos humanos" (1990, p. 26), sino porque los habitantes de las zonas rurales no intervienen en su diseño. Como señala Bustos (2009), las intervenciones educativas en el espacio rural no están protagonizadas por sus habitantes ni responden a un proyecto propio. Por eso, la Educación para el Desarrollo y la Ciudadanía Global (en lo sucesivo, EDCG) resulta de vital importancia en las zonas rurales para despertar actitudes y habilidades en la ciudadanía que permita esclarecer la necesidad de luchar por un mundo más justo (Banks, 2008).

Es en este contexto en el que se enmarca la presente investigación, cuyo propósito principal es el de profundizar, mediante un estudio de carácter correlacional, en el análisis descriptivo efectuado por el proyecto Rural DEAR Agenda EYD-2015 sobre las actitudes de la población rural europea participante en el mismo hacia diferentes cuestiones relativas a la ED.

\section{Educación para el desarrollo y la ciudadanía global}

Explicar qué es la ED resulta complejo debido a que se trata de un concepto ambiguo no exento de críticas y de interpretaciones. Como advierten Camacho, Castellanos y Mayordomo (2010) y Thomson (2016), el significado de ED posee un carácter dinámico debido a los cambios que afectan a la sociedad y al concepto de desarrollo. 
La evolución histórica de la ED se encuentra recogida en el modelo generacional establecido por Mesa (2000). Este sistema generacional incluye inicialmente cinco enfoques y abarcaba desde el origen de la ED, el cual tuvo lugar en los años 40 cuando se intentaba asistir caritativamente a las zonas del Sur tratando de ayudarles a través de recaudaciones de fondos y acciones de sensibilización (Boni y León, 2013), hasta el quinto enfoque que emergió en torno a 1990 bajo la denominación de EDCG o cosmopolita, en el cual se promueve el desarrollo de una ciudadanía solidaria y justa que pretenda transformar el mundo en un lugar mejor. En la actualidad, autores como Calvo (2017) o Sainz de Murieta (2016) afirman que está en vigor una sexta generación que busca "la transformación social necesaria para la construcción de un mundo justo y solidario" (Celorio, 2013, p. 225).

La inexistencia de una definición universal de ED, o de una proposición que esté ampliamente aceptada, hace necesario efectuar una aproximación de lo que se comprende por ED. Con esta finalidad, tomando como base las definiciones recogidas por Bourn (2015), Celorio y Celorio (2011), CONGDE (2008), De la Calle et al. (2003), Marhuenda (2000), Mesa (2000), Ortega (2008, 2010) y Ortega, Cordón-Pedregosa y Sianes (2013), se puede definir la ED de la siguiente forma: proceso educativo que pretende formar personas autónomas y críticas que comprendan los problemas que afectan a la realidad social y a las relaciones entre países del Norte y del Sur, tratando así de crear una ciudadanía global comprometida con la defensa de los Derechos Humanos que transforme la sociedad actual en una más solidaria y justa.

Cabe destacar que, actualmente, se emplea el término de EDCG para referirse a la ED, como se puede apreciar en los estudios de Andreotti (2011), Alcaide y Martínez-Usarralde (2017), Boni (2011, 2012) o Mesa (2014). El concepto de EDCG actual indica la necesidad de promover "valores y actitudes relacionados con la solidaridad y la justicia social. Implica ser un sujeto activo comprometido con los problemas locales y globales, relacionados con la paz, los derechos humanos y la justicia social" (Mesa, 2014, p. 104).

\section{El proyecto europeo Rural DEAR Agenda EYD-20I5}

El proyecto europeo Rural DEAR Agenda-EYD 2015 se centra, por un lado, en estudiar la ED en las zonas rurales de los siguientes países socios: Bulgaria, Chipre, España, Grecia, Italia, Malta y Polonia; y, por otro lado, partiendo de los resulta- 
dos obtenidos, en elaborar una Agenda Rural que recoja propuestas de acciones para impulsar las actuaciones de ED y el Desarrollo Sostenible en zonas rurales (OCUVa, 2016, 2017). En tal Agenda Rural se recogerían diferentes propuestas de actuación que mejorasen la eficacia de las iniciativas impulsadas por las autoridades provinciales y regionales en las zonas rurales europeas con el propósito de "propiciar una mayor conciencia pública, una mejor participación en el desarrollo y una mirada más amplia y solidaria ante otras realidades" (Carracedo, Domenech y Pérez, 2016, p. 12).

El citado proyecto europeo tiene varios objetivos relevantes, entre los que se destaca: sensibilizar a la ciudadanía para que entienda los conflictos relativos a las desigualdades sociales y desarrollar en la población una conciencia global que despierte su sentido de pertenencia al mundo tratando de impulsar un Desarrollo Sostenible y Humano. El proyecto se encuentra fragmentado en cinco fases para poder lograr sus fines, etapas que pueden verse recogidas esquemáticamente en la Figura 1.

FIGURA I. Esquema de las fases del proyecto Rural DEAR Agenda

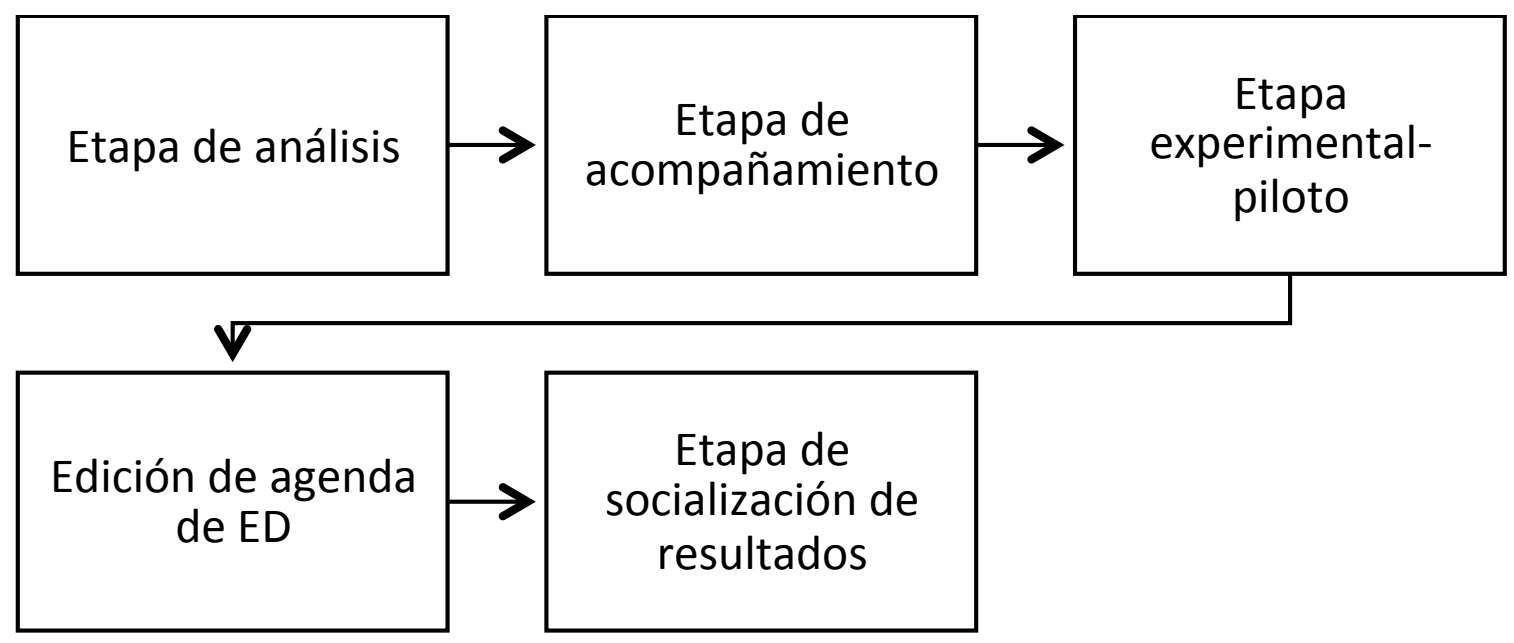

Fuente: elaboración propia.

Por consiguiente, el proyecto pretende ayudar a potenciar una ciudadanía rural europea que atienda a las razones de la solidaridad, las redes de apoyo-ayuda y la responsabilidad compartida para fomentar una Europa más comprometida y sostenible. 


\section{Objetivos}

El objeto de la presente investigación es el de profundizar en el análisis de las respuestas de carácter actitudinal con respecto a la ED recogidas por el proyecto Rural DEAR Agenda-EYD 2015 mediante la identificación de relaciones relevantes o de interés entre las mismas. El cuestionario empleado como instrumento de recogida de información sobre las opiniones de la ciudadanía empleado en el mencionado proyecto pretendía conocer las actitudes y las opiniones de la ciudadanía rural europea sobre diversas cuestiones o problemáticas internacionales, lo que permitió definir un primer perfil actitudinal con respecto a la ED de la población que vive en las zonas rurales europeas que participaron en el proyecto, conformadas por municipios rurales europeos situados en Bulgaria, Chipre, España, Grecia, Italia, Malta y Polonia. Dicho perfil, sin embargo, presentaba un perfil meramente descriptivo que dejaba aún múltiples cuestiones abiertas. Así, la presente investigación pretende dar un paso más en la comprensión de este perfil mediante un primer análisis de posibles relaciones entre las respuestas de los participantes a las que se ha hecho referencia previamente a través de un enfoque de carácter correlacional.

Con base en este objetivo central se establecen los siguientes objetivos específicos, de carácter más operativo:

- Identificar y analizar correlaciones significativas entre las respuestas a los ítems del bloque de preguntas relativo a los intereses informativos y al seguimiento de noticias que realiza la población rural europea sobre temáticas de desarrollo.

- Identificary analizar correlaciones significativas entre las respuestas al bloque de preguntas sobre la contribución personal para mejorar la situación de los países pobres y las correspondientes al bloque de cuestiones relativo a la frecuencia con la que participan en acciones contra la situación de los países en vías de desarrollo.

- Identificar y analizar correlaciones significativas entre las respuestas a los ítems del bloque de cuestiones relacionadas con la frecuencia de participación en acciones que pretenden ayudar a mejorar la situación de los países en desarrollo.

- Identificar y analizar correlaciones significativas entre las diversas respuestas dadas a la cuestión relativa a los motivos que influyen en la ciudadanía rural europea encuestada a la hora de tomar la decisión de participar en acciones para mejorar la situación de los países empobrecidos. 


\section{Metodología}

El presente trabajo se enmarca en un paradigma cuantitativo de aproximación a la realidad a través del uso de un diseño no experimental (Albert, 2007; Pita y Pértegas, 2002) en el que los datos se recopilaron a través del estudio de encuesta establecido en el proyecto Rural DEAR Agenda-EYD 2015; concretamente, se aplicó un cuestionario con preguntas estandarizadas, es decir, formuladas de la misma manera para cada persona encuestada (Cea, 201 1). La encuesta se tradujo a las lenguas oficiales de los países europeos participantes y se proporcionó la posibilidad de cumplimentarlo en papel o electrónicamente.

En cuanto al tipo de análisis llevados a cabo estos se corresponden con análisis de carácter correlacional, los cuales, como indica Kume (1992), permiten juzgar la existencia de correlaciones calculando el coeficiente de correlación o mediante la elaboración de diagramas de dispersión; en particular, el presente estudio se ha centrado en la búsqueda de correlaciones significativas existentes entre las variables que pretendían describir las actitudes de la población rural europea, a través del cálculo del coeficiente de correlación de Pearson. Esta investigación correlacional "tiene en alguna medida, un valor explicativo, aunque parcial. Saber que dos conceptos o variables se relacionan aporta cierta información explicativa" (Gómez, 2006, p.68).

La selección muestral de los participantes que respondieron al cuestionario relativo a la opinión de la población se realizó mediante un diseño muestral basado en un método mixto multi-etapa de muestreo. Por un lado, se aplicó un muestreo de conveniencia por conglomerados que permitió seleccionar los municipios en los que se realizó la encuesta. Se optó por este tipo de muestreo porque resulta útil emplearlo cuando se trata de poblaciones que abarcan una gran área geográfica (Cardona, 2002; Cea, 2001). Por otro lado, se llevó a cabo un muestreo por cuotas con el objetivo de elegir a los individuos participantes. Previamente, se había decidido que las personas participantes debían tener 16 años cumplidos y estar registrados en los censos de las siguientes zonas europeas:

- España: Valladolid (solo la zona rural).

- Italia: Región de Molise.

- Chipre: Municipio de Idalion.

- Grecia: Región de Thessaly.

- Polonia: Región de Lodzkie.

- Bulgaria: Distrito de Lovech.

- Malta: Birgu, Bormia. Isla, Zejtum y Rabat. 
Las cuotas se definieron primordialmente sobre la base de criterios de representatividad fundamentados en la edad y se estableció un tamaño muestral teórico de 2800 participantes, por lo que cada país debió completar 400 cuestionarios, los cuales se encuentran divididos por las cuotas que marcan los rasgos de edad como se puede apreciar en la Tabla 1.

\section{TABLA I. Distribución de la muestra por rango de edad}

\begin{tabular}{|c|c|}
\hline Rango de edad & $\begin{array}{c}\text { Unidades de muestreo } \\
\text { (personas a ser entrevistadas) }\end{array}$ \\
\hline $16-24$ & 120 \\
\hline $25-34$ & 80 \\
\hline $35-44$ & 66 \\
\hline $45-54$ & 54 \\
\hline $55-64$ & 40 \\
\hline $65-74$ & 40 \\
\hline TOTAL & 400 \\
\hline
\end{tabular}

Fuente: elaboración propia.

El número total de cuestionarios cumplimentados fue de 3026, por lo que el tamaño muestral real superó al que se había previsto inicialmente. Esto facilitó "el efecto compensatorio que el tamaño muestral ejerce en un muestreo por cuotas [...], manteniendo por tanto el margen de error muestral establecido para el análisis global" (Marbán y Torrego, 2017, p.53). El error muestral fijado para un nivel de confianza del $90 \%$ y $\mathrm{P}=\mathrm{Q}$ fue de $\pm 5 \%$ para cada país y $\pm 1.8 \%$ para el conjunto de toda la población (Marbán y Torrego, 2017). Al emplear un muestreo aleatorio simple, el tamaño de la muestra teórica calculado para tal supuesto se aumentó en un factor de 1.5 para subsanar el efecto del muestreo por cuotas.

\section{Resultados}

El análisis correlacional objeto de este artículo se llevó a cabo aplicando la matriz de Pearson sobre los ítems de algunas preguntas planteadas en la encuesta de opinión pública y entre bloques de cuestiones; siendo más específicos, el análisis 
se centró en estudiar algunas de las relaciones existentes entre las actitudes de la población rural europea sobre diversas cuestiones ligadas con la ED. En este caso, la realización de estos análisis permite apreciar que hay correlaciones positivas moderadas y altas fuertemente significativas, las cuales se señalan en negrita y con doble asterisco, entre los ítems de una misma pregunta e, incluso, entre los ítems de distintas cuestiones. A continuación, se presenta la Tabla 2 en la que muestra la interpretación, clásica o estándar, por otra parte, para este tipo de investigaciones, del coeficiente de correlación de Pearson.

\section{TABLA 2. Interpretación del coeficiente de correlación de Pearson}

\begin{tabular}{|c|l|}
\hline Valor del coeficiente & \multicolumn{1}{|c|}{ Interpretación } \\
\hline 0 & Correlación nula \\
\hline $0.01-0.19$ & Correlación positiva muy baja \\
\hline $0.20-0.39$ & Correlación positiva baja \\
\hline $0.40-0.69$ & Correlación positiva moderada \\
\hline $0.70-0.89$ & Correlación positiva alta \\
\hline $0.90-0.99$ & Correlación positiva muy alta \\
\hline 1 & Correlación positiva perfecta \\
\hline
\end{tabular}

Fuente: Adaptado de Marbán y Torrego, 2017, p. 69.

Antes de comentar la presentación de resultados de cada uno de los bloques de preguntas analizados, es preciso señalar que son múltiples las correlaciones fuertemente significativas identificadas, con diferentes grados de intensidad, tanto entre diferentes bloques de preguntas, como entre diferentes ítems.

\section{I. Intereses de información y seguimiento de noticias}

La cuestión relativa a los intereses informativos y al seguimiento de noticias que se plantea en la encuesta de opinión pública contiene seis ítems que debían ser valorados en una escala Likert, siendo $1=$ No me importa y $5=$ Seguimiento muy de cerca. La Tabla 3 muestra las correlaciones obtenidas entre los ítems de este bloque de cuestiones. 


\section{TABLA 3. Correlación de Pearson entre las variables sobre el seguimiento de noticias}

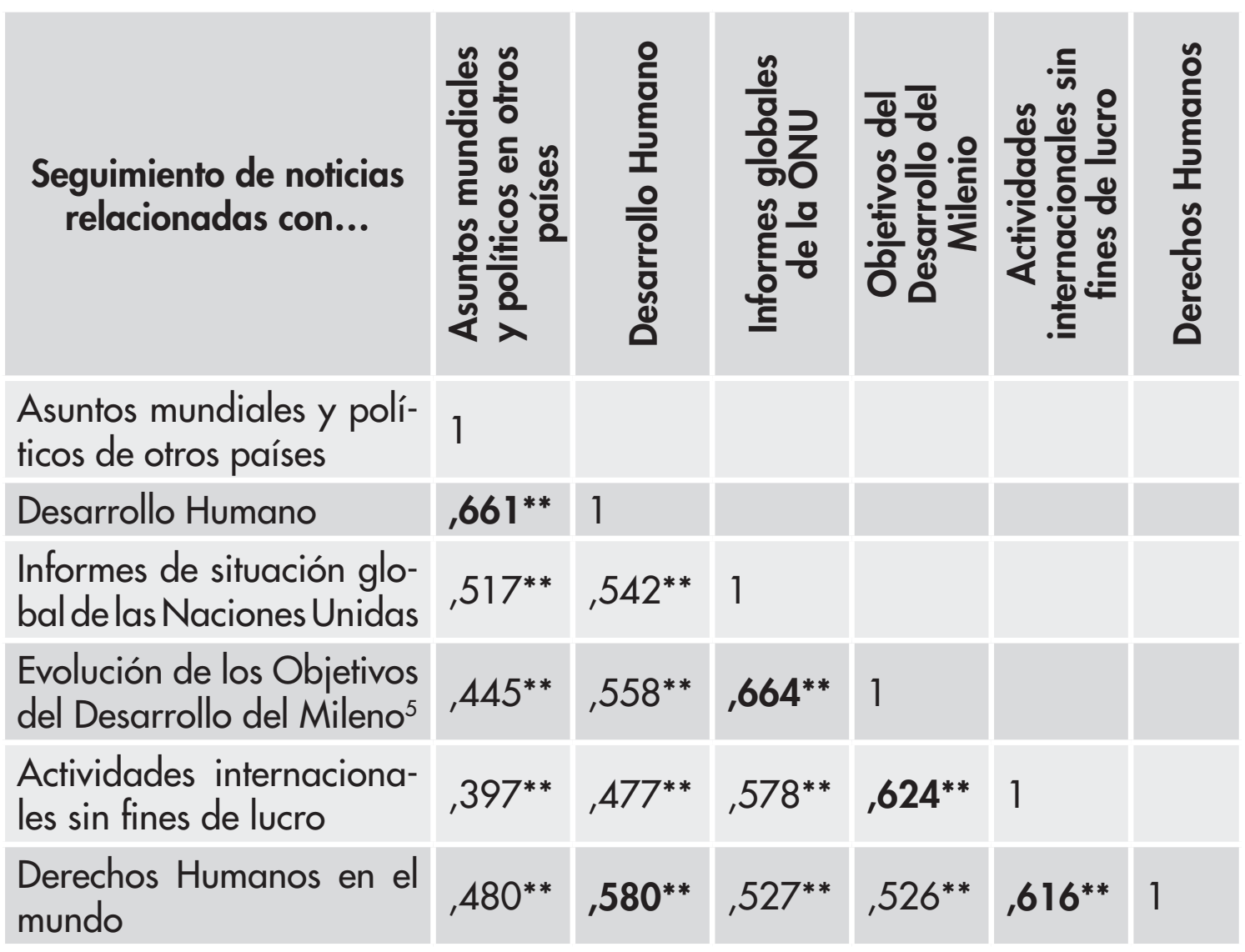

** La correlación es significativa al nivel 0,01 (bilateral).

Fuente: elaboración propia.

En líneas generales, se aprecian en la tabla anterior correlaciones positivas moderadas fuertemente significativas, según la interpretación del coeficiente de correlación de Pearson plasmado en la Tabla 2, entre las diferentes variables que se enmarcan en la cuestión relacionada con el seguimiento de noticias. En este sentido, se evidencian relaciones positivas moderadas entre las personas que realizan un seguimiento de noticias sobre asuntos mundiales y políticos de otros países y las que consultan frecuentemente noticias relacionadas con el Desarrollo

\footnotetext{
${ }^{5}$ A pesar de que los actuales Objetivos del Desarrollo Sostenible estaban en vigor durante esta investigación, se decidió preguntar por los ODM para evitar que pudieran existir dudas o que hubiera algún desconocimiento de los nuevos cambios por parte de los participantes.
} 
Humano (Pearson $=, 661^{* *}$ ). A su vez, la ciudadanía que tiende a mostrar un interés frecuente en noticias sobre Desarrollo Humano también se muestra interesada en cuestiones ligadas a los Derechos Humanos (Pearson=,580"). Estos datos ponen de manifiesto que la ciudadanía rural europea que sigue noticias relacionadas con cuestiones políticas y mundiales a nivel internacional tiende a mostrarse interesada en cuestiones relativas al Desarrollo Humano y a los Derechos Humanos, lo cual muestra una tendencia a estar informada de asuntos sociopolíticos que afectan a todos los países a nivel internacional y, en especial, a los países empobrecidos.

Seguidamente, los resultados presentados en la Tabla 3 permiten observar que el seguimiento de noticias sobre los ODM correlaciona positivamente con el interés por la información sobre la evolución de los informes globales elaborados por la ONU (Pearson $=, 664^{* *}$ ), y las actividades internacionales sin ánimo de lucro (Pearson $\left.=, 624^{* \prime}\right)$. El resto de las correlaciones fuertemente significativas también muestran relaciones positivas moderadas entre el seguimiento de noticias relacionadas con acciones internacionales sin fines de lucro y las cuestiones sobre Derechos Humanos (Pearson=,616"). Estos datos sugieren que la población encuestada interesada en noticias sobre las cuestiones que trata la $\mathrm{ONU}$, entre las que se encuentran los ODM y, por extensión, los Derechos Humanos, tiende a mantenerse informada de las novedades informativas relacionadas con las acciones sin fines de lucro que se realizan a nivel internacional.

\subsection{Contribución personal para mejorar la situación de los países empobrecidos y participación en actuaciones contra la situación de estos países}

Este segundo bloque realiza un análisis que permite apreciar las correlaciones existentes entre la pregunta relativa a la contribución personal para mejorar la situación de los países pobres y el bloque de cuestiones sobre la frecuencia de participación en acciones contra la situación de los países en desarrollo (véase Tabla 4).

La pregunta destinada a conocer si la ciudadanía participante considera que sus acciones pueden contribuir a cambiar la situación de los países empobrecidos está formada por cuatro posibles respuestas siendo $1=S i$, lo intento y $4=$ No creo, es responsabilidad política. Por otra parte, el bloque de preguntas sobre la frecuencia de participación en actividades para ayudar a mejorar la situación de los países empobrecidos contiene siete ítems que debían ser valorados en una escala Likert, siendo $1=$ Nunca y $5=$ Muy a menudo. 


\section{TABLA 4. Correlación de Pearson contribución personal y participación en acciones}

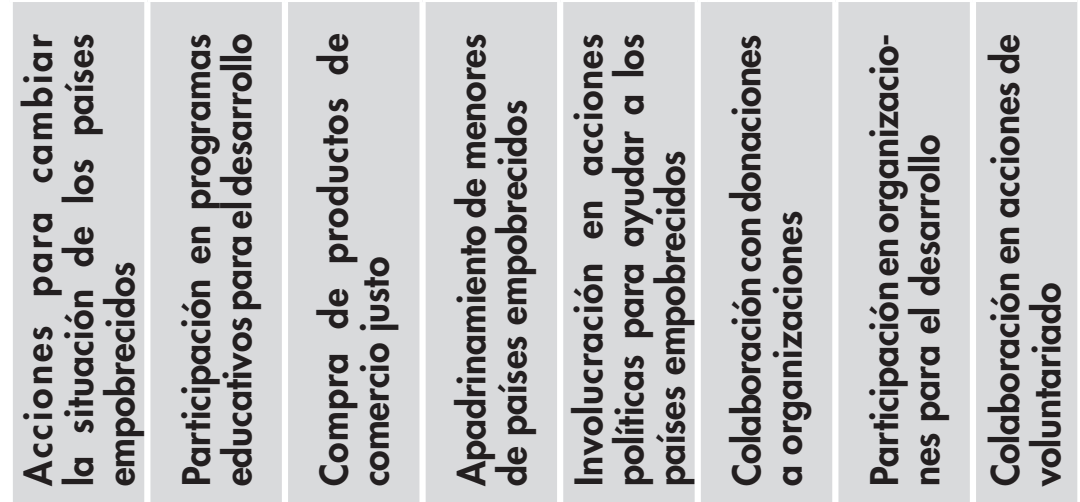

Considera que sus acciones pueden ayudar a cambiar la situación de los países empobrecidos

Frecuencia de participación en programas educativos para el desarrollo

Frecuencia con la que ha comprado productos de comercio justo

Frecuencia con la que ha apadrinado a menores de otros países

Frecuencia con la que se ha involucrado en acciones políticas para ayudar a los países en desarrollo

Frecuencia con la que ha colaborado con donaciones para ayudan a países pobres

Frecuencia con la que ha sido miembro activo de una organización para el desarrollo

Frecuencia en laquehacolaborado en voluntariados

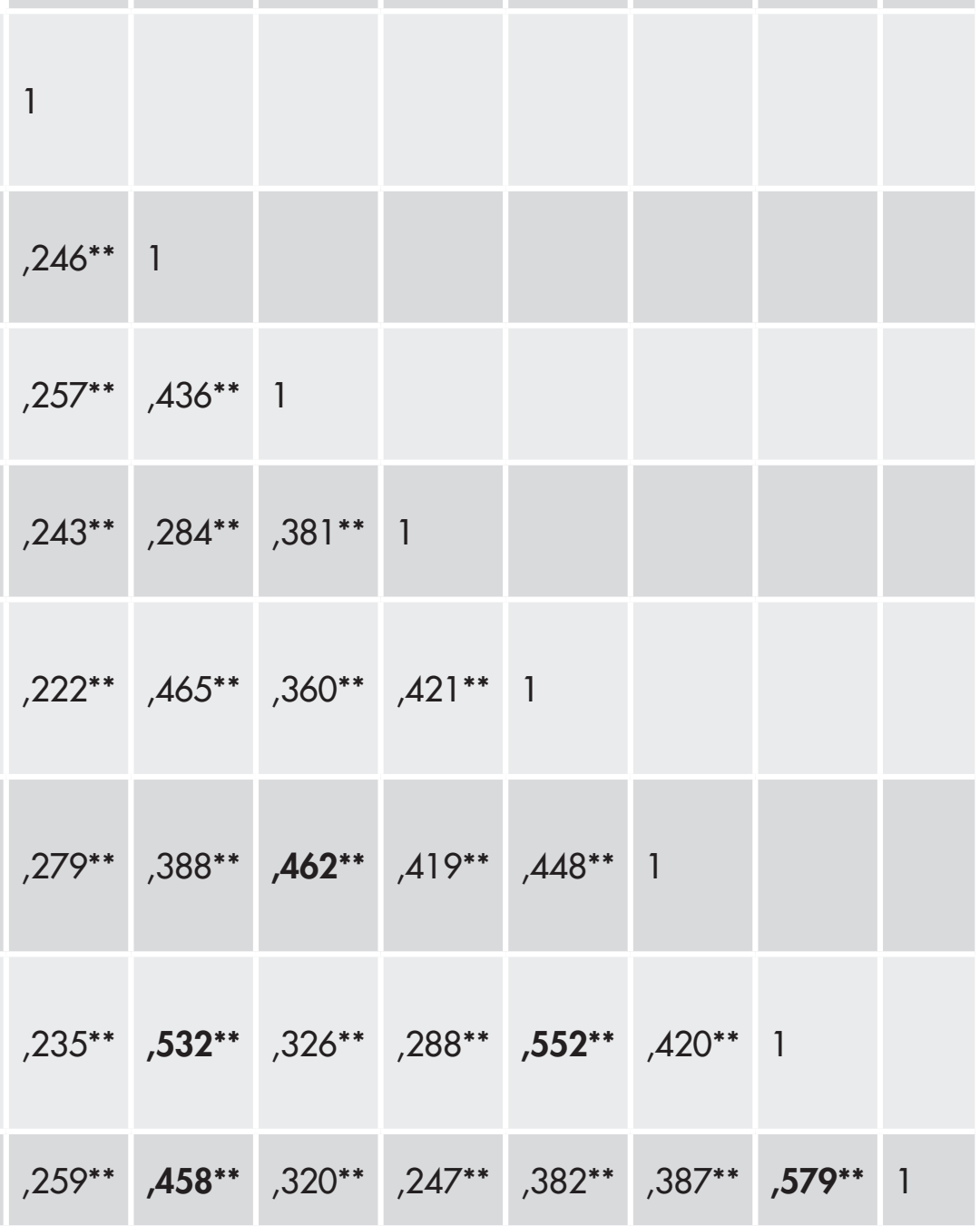

** La correlación es significativa al nivel 0,01 (bilateral).

Fuente: elaboración propia. 
La cuestión centrada en conocer si las personas consideran que sus acciones pueden ayudar a cambiar la situación de los países empobrecidos presenta correlaciones positivas fuertemente significativas con todas las variables relativas a la frecuencia en la que la población europea participa en diversas actividades para mejorar la situación de los más desfavorecidos (véase Tabla 4). Estos datos sugieren que la ciudadanía que considera que puede contribuir personalmente con sus acciones para cambiar la situación de los países empobrecidos tiende a colaborar en actividades de voluntariado (Pearson=,259") y suele realizar frecuentemente donaciones a organizaciones para ayudar a solventar la situación de los países del Sur (Pearson=,279"); por tanto, se puede interpretar que cuando la ciudadanía está sensibilizada y es consciente de la importancia que poseen sus acciones para ayudar a cambiar la situación de los países empobrecidos, actúa fundamentalmente a través de donaciones y participando en actividades puntuales de voluntariado.

Los resultados obtenidos en la cuestión sobre la frecuencia de participación de los encuestados en una serie de acciones propuestas muestran la existencia de correlaciones positivas moderadas entre todos los ítems de esta cuestión. Por un lado, los resultados permiten observar que la ciudadanía rural europea que ha participado en programas educativos de desarrollo tiende a ser miembro de organizaciones para el desarrollo (Pearson $=, 532^{* *}$ ) y a involucrarse en acciones políticas para ayudar a los países empobrecidos (Pearson $=, 552^{* *}$ ). Estos resultados indican que si la ciudadanía está correctamente formada ante las cuestiones globales propias de la EDCG tiende a ir más allá de realizar meras donaciones o acciones puntuales, pues participan con relativa frecuencia en acciones políticas y son miembros de organizaciones cuyo propósito es ayudar a los países empobrecidos.

Adicionalmente, los resultados presentados en la Tabla 4 permiten observar correlaciones positivas fuertemente significativas entre la población europea encuestada que frecuentemente compra productos de comercio justo y la colaboración con donaciones a organizaciones (Pearson $=, 462^{* *}$ ). Estas relaciones sugieren que las personas que colaboran mediante donaciones puntuales tienden entre sus acciones a comprar productos de comercio justo para tratar de ayudar a los países empobrecidos, lo cual sugiere la necesidad de formar ciudadanos globales críticos se involucren activamente a favor de la justicia social y no se queden relegados realizar meras donaciones de fondos. En caso contrario, la población encuestada que actúa y participa, tal como se pretende conseguir mediante una adecuada educación para el desarrollo, en cuestiones políticas para ayudar a los países empobrecidos es la que presenta más frecuencia en el apadrinamiento de niños de dichos países (Pearson=,421"). 
6.3. Valoración de la influencia de las acciones para solventar la situación de los países empobrecidos

Tras analizar en el subapartado anterior las correlaciones existentes en la participación de la ciudadanía en acciones para mejorar la situación de los países en vías de desarrollo, se procede ahora a plasmar las correlaciones existentes entre los motivos que llevan a los encuestados a participar en dichas labores. De forma previa, se debe señalar que este bloque de preguntas contiene cinco ítems que son valorados en una escala Likert, siendo $1=$ Ninguna y $5=$ Muy alta.

\section{TABLA 5. Correlación de Pearson influencia de las acciones para mejorar la situación de los países empobrecidos}

\begin{tabular}{|c|c|c|c|c|c|}
\hline $\begin{array}{l}\text { Influencia de las siguientes ra- } \\
\text { zones en su decisión de unirse } \\
\text { a las acciones marcadas ... }\end{array}$ & 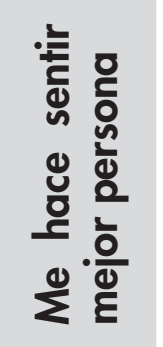 & 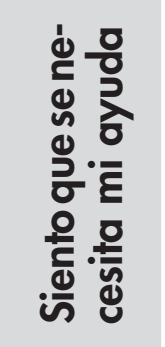 & 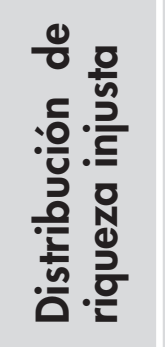 & 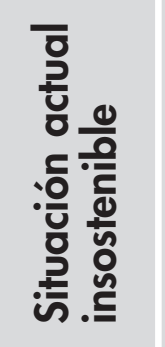 & 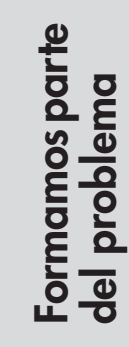 \\
\hline $\begin{array}{l}\text { Porque me hace sentir mejor } \\
\text { persona }\end{array}$ & 1 & & & & \\
\hline $\begin{array}{l}\text { Porque siento que se necesita mi } \\
\text { ayuda }\end{array}$ &, $682^{* *}$ & 1 & & & \\
\hline $\begin{array}{l}\text { Porque la distribución de riqueza } \\
\text { no es justa }\end{array}$ &, $448^{* *}$ &, $522^{* *}$ & 1 & & \\
\hline $\begin{array}{l}\text { Porque la situación actual no es } \\
\text { sostenible }\end{array}$ &, $377^{* *}$ & $447^{* *}$ & ,688** & 1 & \\
\hline Porque somos parte del problema &, $344^{* *}$ &, $396^{* *}$ &, $516^{* *}$ &, $636 * *$ & 1 \\
\hline
\end{tabular}

La Tabla 5 muestra las correlacionales positivas, todas ellas fuertemente significativas, existentes entre las diferentes preguntas que pretendían conocer qué influye en las personas para que participen en actividades relacionadas con la ED ya nombradas en la Tabla 4. Los resultados muestran que las personas que participan 
en esas acciones para sentirse mejor persona también tienden a hacerlo porque piensan que su ayuda es necesaria (Pearson=,682"). Estos resultados sugieren que las personas participantes que actúan únicamente para sentirse mejor persona buscan mostrarse satisfechos consigo mismos al ofrecer una ayuda que consideran necesaria para mejorar la situación de los países empobrecidos.

En cuanto a las personas encuestadas que actúan porque comprenden que la distribución de riqueza es injusta suele también participar al considerar que la situación actual es insostenible (Pearson=,688") y porque se conciben como parte del problema (Pearson=,636"). Estos resultados ponen en relieve que la ciudadanía encuestada que realmente está comprometida y comprende la necesidad de actuar para cambiar las injusticias sociales actuales, las cuales solamente generan desigualdades especialmente en los países empobrecidos, se implica en las acciones ya señaladas no solamente para sentirse mejor persona, sino por algo mucho más importante: considerarse parte del problema y entender la necesidad de transformar la sociedad en una más sostenible y justa para todos.

\section{Discusión}

Los resultados obtenidos sobre los intereses de información y el seguimiento de noticias de la población rural europea encuestada desvelan diversas relaciones entre los factores analizados. De esta forma, se puede apreciar que la población rural europea que muestra interés por las noticias relacionadas con los asuntos mundiales y políticos de otros países, también se involucra en las cuestiones sobre el Desarrollo Humano. Autores como Boni, Belda-Miquel y Pellicer-Sifres (2018) o Fajardo (2015) se muestran de acuerdo en que el Desarrollo Humano ha ido despertando interés a raíz de estudios como los publicados por Sen (1999) o Nussbaum (2012); además, el Programa de Naciones Unidas para el Desarrollo (PNUD) se ha encargado desde 1990 de ir elaborando informes sobre esta cuestión. Otros autores, como Beutelspacher y Zapata (2015), señalan a las investigaciones sobre cuestiones de género como punto clave para despertar este interés que se aprecia en la población sobre las noticias relativas al Desarrollo Humano; concretamente, a juicio de estos autores, actualmente se está promoviendo un cambio hacia la noción de Desarrollo Humano Sostenible, pues resulta necesario garantizar el bienestar, la libertad y la equidad de todos los ciudadanos.

Seguidamente, como podría esperarse, la ciudadanía que se muestra interesada en noticias de Desarrollo Humano y sobre la necesidad de garantizar el bienestar y la 
igualdad realiza un fuerte seguimiento en cuestiones relacionadas con los Derechos Humanos. Este interés de la población rural europea encuestada en cuestiones sobre los Derechos Humanos se puede relacionar con su indiscutible importancia a nivel global y por el interés que se ha despertado en Europa desde hace años para velar por su protección garantizando así el bienestar social (Mangas, 2018).

Los Derechos Humanos han ido cobrando cada vez más presencia en las agendas de todos los países, al ser la base de iniciativas tan relevantes como los actuales Objetivos del Desarrollo Sostenible (ONU, 2015); incluso, los anteriores objetivos mundiales, denominados Objetivos del Desarrollo del Milenio (ODM), ya se encontraban vinculados con los Derechos Humanos (Verdiales, 2018). Como consecuencia de esto, parece claro intuir que la población interesada en noticias sobre los informes internacionales elaborados por la ONU también realiza un seguimiento de las noticias sobre los ODM, tal como se ha comprobado, y éstas, a su vez, por las noticias que tratan sobre actividades internacionales sin ánimo de lucro. Por consiguiente, se aprecia que la ciudadanía que muestra interés en dichas actividades también lo hace por las noticias sobre los ODM y los Derechos Humanos.

Respecto a las resultados obtenidos en la contribución personal de la población europea participante para mejorar la situación de los países empobrecidos y en actuaciones contra la situación de estos países, los datos muestran que la ciudadanía encuestada considera que sus acciones pueden favorecer el cambio de la situación de los países en desarrollo; no obstante, estas acciones tienden a realizarse especialmente a través de donaciones dedicadas a la ayuda de los problemas de estos países y colaborando en actividades de voluntariado. Esto indica que las actuaciones que realizan para favorecer el cambio parecen no requerir una involucración activa, sino que tienden a quedarse reducidas a meras donaciones de fondos o participaciones puntuales en acciones propuestas por diversas organizaciones. En esta línea, Jáuregui (2018) señala que la Unión Europea "es el mayor donante de ayuda al desarrollo y humanitaria en el mundo, [...] lidera la lucha contra el cambio climático y su diplomacia contribuye a aumentar la seguridad y la sostenibilidad mundiales" (p.71). Sin embargo, se debe añadir que una ciudadanía global formada adecuadamente no puede solamente quedarse en ser sensibilizada y realizar donativos, pues actualmente se necesita una ciudadanía global crítica que actúe en defensa de la justicia social (Andreotti, 2006).

A su vez, la ciudadanía encuestada que afirma participar frecuentemente en programas educativos de desarrollo también tiende a colaborar con organizaciones de desarrollo y éstas, a su vez, tienden a participar en acciones políticas o de 
voluntariado. La educación posee el papel más relevante para formar ciudadanos globales $y$, por tanto, se puede suponer que si las personas poseen una sólida formación van a tender a participar en acciones políticas o de voluntariado para defender los Derechos Humanos y la justicia social. Concretamente, entre los objetivos de la EDCG se puede destacar: la formación de una ciudadanía crítica que se implique en "acciones de movilización social e incidencia política" (Celorio, 2013, p.241). Esta formación en EDCG, como indican Argibay y Celorio (2005), puede provenir tanto del ámbito formal (centros de enseñanza reglada), como del no formal (enseñanzas extraescolares, escuelas de adultos...) y del informal (medios de comunicación, campañas informativas...).

Adicionalmente, se aprecian dos correlaciones fuertemente significativas relevantes en función de las actuaciones contra la situación de los países empobrecidos por parte de los participantes; por un lado, la ciudadanía que suele apadrinar a menores de otros países tiende a participar frecuentemente en actividades políticas para ayudar a los países empobrecidos; $y$, por otro lado, las personas que compran productos de comercio justo suelen realizar donaciones para ayudar a los países en desarrollo. En este sentido, la ciudadanía concienciada que se promueve desde la EDCG permite demostrar que esta correlación es fruto de la responsabilidad ciudadana que se intenta inculcar (Doppler y González, 2007); de este modo, los consumidores buscan comprar productos de comercio justo para satisfacer así sus valores éticos y fomentar un Desarrollo Sostenible (Lipovetsky, 2005). No obstante, los gobiernos también tienen un papel importante a la hora de ayudar a los países empobrecidos porque deben mostrarse más involucrados en promover un comercio más justo y equitativo entre los países del Norte y los del Sur (Cantos, 1998).

Finalmente, respecto a los resultados relativos a la valoración de la influencia de las acciones para solventar la situación de los países empobrecidos, la ciudadanía rural europea participa en las actuaciones ya comentadas en la Tabla 2 por diversos motivos; entre ellos, hay personas que actúan porque les hace sentir mejor persona, suelen también actuar porque sienten que se necesita su ayuda. Torroja (1998) señala que las personas que comprenden la importancia de la cooperación internacional poseen una sólida formación en valores que les permite ser solidarios y colaborar en acciones de ayuda al desarrollo, aunque la mayoría de los problemas no tienen fácil solución al tener implicados múltiples factores relacionados con las políticas de los Estados.

En línea con lo anterior, los resultados revelan que una gran parte de la ciudadanía considera que debe actuar porque se necesita su ayuda y su participación 
tiende a estar influida por la injusta distribución de riqueza que se puede apreciar en la sociedad actual; de esta forma, la población que actúa debido a la injusta repartición de riquezas existente suele conducirse también bajo la influencia de la insostenibilidad de la situación actual e, incluso, porque se perciben a sí mismos como parte del problema. De esta forma, las personas que se consideran parte del problema muestran un elevado grado de responsabilidad por lo que suelen colaborar tratando de mejorar la insostenible situación actual. Autores como Meira (2006) y Delgado (2016) afirman que esta insostenibilidad es certera, pues es necesario que se produzca un cambio radical hacia una "nueva ecología políitica donde todos los humanos, etnias y culturas podamos convivir respetándonos mutuamente, repartiendo igualitariamente los bienes producidos y practicando una democracia participativa" (Delgado, 2016, p.64).

\section{Conclusiones}

Los diferentes análisis correlacionales presentados en este estudio permiten concluir que hay múltiples relaciones no evidentes a simple vista o de forma intuitiva entre las preguntas de los diferentes bloques de cuestiones analizados. De esta forma, se aprecia una relevante relación en todas las cuestiones estudiadas que merece ser analizada en profundidad, incluso en algunos casos en términos de posibles relaciones de carácter causal, estableciendo análisis predictivos.

En cuanto a los objetivos propuestos inicialmente, a través de este estudio se ha logrado establecer la existencia de múltiples correlaciones positivas fuertemente significativas entre las preguntas del cuestionario de opinión pública que tratan sobre las actitudes de la población rural europea ante diversas cuestiones de ED. Cabe añadir que los objetivos específicos se han cumplido pudiendo señalar diversas consideraciones:

- Existen correlaciones positivas fuertemente significativas entre los ítems relativos al seguimiento de noticias, el cual ha permitido apreciar, por ejemplo, que la ciudadanía que realiza un seguimiento sobre la evolución de los ODM también muestra interés por las novedades relativas a los informes internacionales que realiza la ONU.

- Las correlaciones entre la contribución personal que realizan las personas encuestadas para mejorar la situación de los países empobrecidos y la frecuencia con la que participan en acciones para mejorar la situación de dichos países 
permiten apreciar que las acciones de la ciudadanía rural europea tienden a quedarse limitadas a recaudaciones de fondos o colaboraciones puntuales en acciones promovidas por organizaciones de desarrollo.

- Los ítems del bloque de cuestiones relacionadas con la frecuencia de participación en acciones que pretenden ayudar a mejorar la situación de los países empobrecidos presentan correlaciones positivas significativas permitiendo apreciar, por ejemplo, que la ciudadanía rural europea que suele participar en actuaciones de voluntariado también tiende a colaborar con las organizaciones para el desarrollo.

- Las respuestas obtenidas sobre los motivos que incitan a la participación ciudadana en acciones para ayudar a mejorar la situación de los países empobrecidos presentan fuertes correlaciones significativas; por ejemplo: la ciudadanía que piensa que la situación actual no es sostenible tiende a actuar también porque considera que el reparto de riquezas actual es injusto.

En resumen, gracias a la realización de este estudio correlacional se han podido apreciar conexiones que permiten intuir cómo suelen proceder las personas que viven en zonas rurales europeas y algunas de las relaciones existentes entre: las noticias de desarrollo que suelen consultar, su contribución personal ante la mejora de la situación de los países en desarrollo, la frecuencia con la que participan en acciones para el desarrollo y las razones que les incitan a tomar la decisión colaborar en acciones para mejorar la situación de los países empobrecidos.

Cabe añadir que los resultados obtenidos muestran indicios de que la población está sensibilizada pero no se implica de forma profunda en cuestiones relativas al desarrollo, lo que permite apreciar que sigue siendo necesario seguir avanzando en el diseño de estrategias efectivas de ED en estas zonas rurales europeas. Una correcta EDCG no debe tener como único objetivo la sensibilización, sino que debe: sensibilizar, formar, concienciar y movilizar (Ortega, 2007).

Por consiguiente, se considera que el presente estudio aporta una visión global complementaria a la meramente descriptiva sobre las actitudes que presenta la ciudadanía rural europea muy útil para comprender mejor cómo tienden a actuar ante temáticas ligadas a cuestiones de desarrollo. 


\section{Bibliografía}

AlbeRT, M. J. (2007). La investigación educativa: claves teóricas. Madrid: McGraw-Hill.

Alcaide, A., y Martínez-Usarralde, M. J. (2017). "La Educación para el Desarrollo (EpD) en un mundo diverso. Estudio comparativo de la Educación para la Ciudadanía Global (EpCG) en Paraguay y España". Quaderns electrònics sobre el desenvolupaments humà i la cooperació, 7, p. 19-36.

ANDREOTI, V. (2006). "Soft versus critical global citizenship education". Policy \& Practise, 3(1), 40-51.

- (2011). "(Towards) decoloniality and diversality in global citizenship education". Globalisation, Societies and Education, 9 (3-4), pp. 381-397.

Argibay, M., y Celorio, G. (2005). La Educación para el Desarrollo. Vitoria: Gobierno Vasco.

BANKS, J. (2008). "Teaching for Social Justice, Diversity, and Citizenship in a Global World". Educational Forum, 68 (4), pp. 296-305.

Beutelspacher, A. N., y Zapata, E. (2015). "Desarrollo, bienestar y género: consideraciones teóricas". Revista de Estudios de Género, La Ventana, 1(1 1), 73-119.

Bonl, A. (2011). "Educación para la ciudadanía global. Significados y espacios para un cosmopolitismo transformador", Revista Española de Educación Comparada, (17), pp. 65-85. DOI: http://dx.doi.org/10.5944/reec.17.2011.7545.

- (2012). "Educando para la ciudadanía global. Una experiencia de investigación cooperativa entre docentes y profesionales de las ONGD", Estudios sobre educación, 23, pp. 63-81.

Bonl, A., Belda-Miquel, S., y Pelicer-Sifres, V. (2018). "Innovación transformadora. Propuestas desde la innovación social colectiva para el desarrollo humano". Recerca, Revista de Pensament i Anàlisi, 23, 67-94.

BONI, A., y LEÓN, R. (2013). "Educación para una ciudadanía global: una estrategia imprescindible para la justicia social". Informe Intermón Oxfam: La Realidad de La Ayuda 2012, pp. 215-239. 
BOURN, D. (2015). The Theory and Practice of Development Education: A pedagogy for global social justice. Nueva York: Routledge.

Bustos, A. (2009). "La escuela rural española ante un contexto en transformación". Revista de Educación, 350, 449-461.

Calvo, A. (2017). "The state of development education in Spain: Initiatives, trends and challenges". International Journal of Development Education and Global Learning, 9(1), pp. 18-32.

Camacho, P., Castellanos, E., y Mayordomo, P. (2010): Educación para el Desarrollo. Una vía hacia la transformación, Cruz Roja Juventud, Madrid.

CANTOS, E. (1998). El porqué del comercio justo: hacia unas relaciones Norte-Sur más equitativas. Barcelona: Icaria.

CARDONA, M. C. (2002). Introducción a los Métodos de Investigación en Educación. Madrid: EOS.

Carracedo, M., Domenech, E., y Pérez, L. (2016). Marco introductorio. En OCU$\mathrm{Va}$, Diagnóstico de la Educación para el Desarrollo en el ámbito rural europeo (pp.12-17). Observatorio Internacional para el Desarrollo de la Universidad de Valladolid (OCUVa), Valladolid.

CEA, M. A. (2001). Metodología cuantitativa: estrategias y técnicas de investigación social. Síntesis, Madrid.

Celorio, G. (2013). Sensibilización y Educación para el Desarrollo. En M. Agost, A. Fuertes, I. Giménez, y G. Soto (Coords.), Cooperación descentralizada pública Introducción, enfoques y ámbitos de actuación (pp.225-261). Castelló de la Plana: Publicaciones de la Universidad Jaume I.

Celorio, J. J., y Celorio, G. (201 1). "¿Educación? para el ¿̇desarrollo?" Pueblos. Revista de información y Debate, 46, 1-3.

Coordinadora de ONGD España (CONGDE). (2008). Código de conducta de las ONG de desarrollo. Madrid: CONGDE.

De la calle, M. J., Rodríguez, M., Ruíz, E., y Torrego, L. (2003). La Educación para el Desarrollo en el marco educativo. Valladolid: GEDEPAZ. Departamento de Didáctica y Organización Escolar Facultad de Educación. Universidad de Valladolid. 
DelGado, L. (2016). "La necesaria convergencia entre el norte y el sur". Sustentabilidad(es), 7 (13), $63-95$.

DOPPLER, F., y GONZÁLEZ, A. A. (2007). "El comercio justo: entre la institucionalización y la confianza". Problemas del desarrollo, 38(149), 181-202.

EkANAYAKE, S. B. (1990). "La pedagogía en zonas rurales: fomentas desde un principio el desarrollo rural. Perspectivas". Revista trimestral de educación comparada, (1). 127-141.

FaJARDO, L. (2015). "Desarrollo Humano Sustentable: Concepto y Naturaleza". Revista electrónica de difusión científica, 10, 1-30.

GómEZ, M. M. (2006). Introducción a la metodología de la investigación científica. Córdoba: Brujas.

JÁUREGUI, R. (2018). "El futuro de Europa (o más bien la Europa del futuro)". Revista de Fomento Social, 73(289), 65-90.

Kume, H. (1992). Herramientas estadísticas básicas para el mejoramiento de la calidad. Barcelona: Norma.

LIPOVETSKY, G. (2005). El crepúsculo del deber. La ética indolora de los nuevos tiempos democráticos. Barcelona: Anagrama.

MANGAS, A. (2018). Unión Europea: Derechos Humanos y Desarrollo Sostenible. En Díaz y Fernández (Eds.), Objetivos de Desarrollo Sostenible y Derechos Humanos: paz, justicia e instituciones sólidas / Derechos Humanos y empresas (pp.13-26). Madrid: Universidad Carlos III.

Marbán, J. M. y ToRrego, L. M. (2017). Estudio de la opinión pública. En OCUVa, Análisis de la Educación para el Desarrollo en el ámbito rural europeo (pp.47-71). Valladolid: Observatorio Cooperación Internacional para el Desarrollo de la Universidad de Valladolid (OCUVa).

Marhuenda, F. (2000). "Solidaridad y Educación para el Desarrollo". Comunicar, $15,93-98$.

MESA, M. (2000). "La educación para el desarrollo: entre la caridad y la ciudadanía global". Papeles de cuestiones internacionales, 70, 11-26. 
MESA, M. (2014). "Precedentes y evolución de la Educación para el Desarrollo: un modelo de cinco generaciones". Sinergias - Diálogos Educativos Para a Transformação Social, (1), 24-56.

MEIRA, P. (2006). "Crisis ambiental y globalización: Una lectura para educadores ambientales en un mundo insostenible". Trayectorias, 8(20-21), 110-123.

Nussbaum, M. (2012). Las mujeres y el desarrollo humano. Barcelona: Herder Editorial.

ObSERVATORIO de COOPERACIÓN INTERNACIONAL PARA El DESARROLlO de LA UNIVERSIDAD DE VALLADOUD (OCUVa). (2016). Resumen ejecutivo del diagnóstico de la Educación para el Desarrollo en el ámbito rural europeo: resumen ejecutivo. Valladolid: OCUVa.

- (2017). Análisis de la Educación para el Desarrollo en el ámbito rural europeo. Valladolid: OCUVa.

ORgANIZACIÓN DE LAS NACIONES UNIDAS (ONU). (2015). Transformar nuestro mundo: la Agenda 2030 para el desarrollo sostenible. Recuperado de: http://www.un.org/ ga/search/view_doc.asp? symbol=A/RES/70/1\&Lang=E.

ORTEGA, M. L. (2007). Estrategia de Educación para el Desarrollo de la Cooperación Española. Madrid: Ministerio de Asuntos Exteriores y de Cooperación. Secretaría de Estado de Cooperación Internacional Dirección General de Planificación y Evaluación de Políticas para el Desarrollo.

- (2008). "La Educación para el Desarrollo: dimensión estratégica de la cooperación española". Cuadernos internacionales de tecnología para el desarrollo humano, 7, 15-23.

- (2010). I Premio Nacional de Educación para el Desarrollo «Vicente Ferrer». Madrid: Asociación Española de Cooperación Internacional para el Desarrollo, Ministerio de Asuntos Exteriores y de Cooperación.

Ortega, M. L., Cordón-Pedregosa, M. R. y Sianes, A. (2013). "La Educación para el Desarrollo en el espacio universitario: de la formación en contenidos a la formación integral". Revista Española del Tercer Sector, 25, 53-78.

PitA, S., y PÉRTEGAS, S. (2002). "Investigación cuantitativa y cualitativa". Cad Aten Primaria, 9, 76-78. 
SAINZ DE MURIETA, J. (2016). “El papel de las Enseñanzas Técnicas Universitarias en la Cooperación Universitaria al Desarrollo y en la formación de ciudadanía global y transformadora". IKASTORRATZA. e-Revista de Didáctica, 17, 1-7.

SEN, A. (1999). Development as Freedom. Oxford: Oxford University Press.

THOMPSON, K. (2016). "Developing Education v. Education for Development". ANNALS, AAPSS, 424, 16-28.

TORROJA, H. (1998). "La relación entre la asistencia humanitaria y la ayuda al desarrollo en la práctica de la ONU". Revista cidob d'afers internacionals, 40/41, 109-133.

VERDIALES, M. (2018). La importancia del enfoque de los derechos humanos en los Objetivos de Desarrollo del Sostenible. En Díaz y Fernández (Eds.), Objetivos de Desarrollo Sostenible y Derechos Humanos: paz, justicia e instituciones sólidas / Derechos Humanos y empresas (pp. 75-90). Madrid: Universidad Carlos III. 